\title{
Evidence from Molecular Markers and Population Genetic Analyses Suggests Recent Invasions of the Western North Pacific Region by Biotypes B and Q of Bemisia tabaci (Gennadius)
}

\author{
CHIA-HUNG HSIEH, CHUNG-HSIUNG WANG, AND CHIUN-CHENG KO ${ }^{1}$ \\ Department of Entomology, National Taiwan University, Taipei 106, Taiwan
}

Environ. Entomol. 36(4): 952-961 (2007)

\begin{abstract}
Invasive events by Bemisia tabaci (Gennadius) biotypes in various parts of the world are of continuing interest. The most famous is biotype $\mathrm{B}$ that has caused great economic losses globally. In addition, biotype $\mathrm{Q}$ has also recently been reported to be a new invasive pest. These two biotypes have been monitored for some time in the Western North Pacific region, but the invasive events and population genetic structures of these two biotypes are still not clear in this region. In this study, the mitochondrial cytochrome oxidase I (COI) gene was used to reconstruct a phylogenetic tree for identifying biotypes $\mathrm{B}$ and $\mathrm{Q}$ and to study the relationships between invasive events and ornamental plants. Population genetic analyses of mtCOI sequences were also used to study the genetic relationships within and between populations. A combination of a phylogenetic tree and haplotype analysis suggested the recent invasion of biotype $\mathrm{Q}$ in this region is related to the international ornamental trade from the Mediterranean region. Low levels of haplotype diversity and nucleotide diversity indicate that the presence of biotypes B and Q in the Western North Pacific region are caused by multiple invasions. Hierarchical analysis of molecular variance supports the hypothesis of multiple invasions. In addition, high sequence identities and low genetic distances within and between populations of the two biotypes revealed that these invasive events occurred recently. The low levels of genetic differentiation revealed by pairwise $F_{\mathrm{ST}}$ values between populations also suggests the invasions were recent. Therefore, results of this study suggested that biotypes B and Q entered this region through multiple recent invasions. A quarantine of agricultural crops may be necessary to prevent further invasions.
\end{abstract}

KEY WORDS whitefly, molecular marker, phylogeny, population genetics, pest

The whitefly, Bemisia tabaci (Hemiptera: Aleyrodidae), was originally described as Aleyrodes tabaci by Gennadius in 1889 on tobacco in Greece (Perring 2001). This species is an important agricultural pest and causes great economic losses (Brown et al. 1995, De Barro 1995, Perring 2001). B. tabaci is widely distributed throughout tropical and subtropical areas of the world (Brown et al. 1995). The concept of biotypes of B. tabaci, proposed in the $1950 \mathrm{~s}$, indicates that morphologically indistinguishable populations exhibit different biological traits (Brown et al. 1995). Variations in biotypes exist in terms of host range, dispersal behavior, fecundity, insecticide resistance, and transmission competency for begomoviruses (Berry et al. 2004).

More than 24 biotypes of $B$. tabaci have been identified by multiple techniques (Perring 2001), the most well-known being the biotype B superbug. It is polyphagous with a broad host range and causes damage through feeding, excretion of honeydew, and virus transmission (De Barro 1995). Biotype B was de-

${ }^{1}$ Corresponding author, e-mail: kocc2501@ntu.edu.tw. scribed previously as B. argentifolii Bellows and Perring, with the proposed common name of silverleaf whitefly (Perring 2001). Biotype Q was originally though to be restricted to the Iberian Peninsula but recently has been widely reported in the Mediterranean Basin (Horowitz et al. 2005, De la Rúa et al. 2006). Horowitz et al. (2005) indicated that biotype Q has a high level of resistance to insecticides and also causes economic damage. Furthermore, biotypes B and Q of B. tabaci have different inherent levels of resistance to insecticides, and insecticide applications affect the proportion of both biotypes (Horowitz et al. 2005). Molecular markers have been developed to identify biotypes B and Q and to study their population dynamics (Khasdan et al. 2005).

Molecular markers are useful tools for distinguishing biotypes and include esterase, random amplified polymorphic DNA polymerase chain reaction (RAPDPCR), amplified fragment length polymorphism (AFLP), and mitochondrial, ribosomal, and microsatellite DNA markers (De Barro and Driver 1997, Frohlich et al. 1999, De Barro et al. 2000, De Barro 2005, Zhang et al. 2005). Based on the genetic diversity 
revealed by RAPD-PCR analysis, biotype B is likely an invader, and biotype $\mathrm{Q}$ may be endemic to the Iberian Peninsula (Moya et al. 2001). In addition, many reports have used mitochondrial and ribosomal markers to reconstruct phylogenetic trees and distinguish biotypes based on tree topology (Frohlich et al. 1999, De Barro et al. 2000, Abdullahi et al. 2003, De la Rúa et al. 2006, Hsieh et al. 2006, Ueda and Brown 2006). Accordingly, biotype $\mathrm{B}$ presumably originated in the Middle East, and evidence supports its having spread worldwide because of human trade activities (Frohlich et al. 1999, De Barro et al. 2000).

The phylogenetic tree of the mitochondrial cytochrome oxidase I ( $\mathrm{mtCOI}$ ) gene indicated four biotypes of B. tabaci in East Asia: B, Q, Nauru, and An (Hsieh et al. 2006). The Nauru and An biotypes are indigenous to East Asia (Hsieh et al. 2006). Biotype B as an invader is the most widely distributed biotype in the Western North Pacific region, and it has already caused huge economic losses in the region (Zhang et al. 2005, Hsieh et al. 2006, Ueda and Brown 2006). Biotype $\mathrm{Q}$ was previously found only in China, but now has also been reported from Japan (Zhang et al. 2005, Ueda and Brown 2006). Otherwise, biotype Q has been reported only in a local region of China and Japan (Zhang et al. 2005, Ueda and Brown 2006). Some reports have hypothesized that ornamental plants such as the poinsettia may have been a possible vector of entry for biotypes B and Q into China and Japan (Zhang et al. 2005, Ueda and Brown 2006). However, the relationships between invasive events and ornamental plants are indefinite, and genetic relationships within and between populations also are not clear in the Western North Pacific region.

Mitochondrial DNA is haploid and particularly useful for population studies because it undergoes no recombination, is maternally inherited, and has a simple sequence organization (Harrison 1989). In addition, the mtCOI gene has been a popular marker for studying relationships among $B$. tabaci biotypes (Frohlich et al. 1999, De la Rúa et al. 2006, Hsieh et al. 2006, Ueda and Brown 2006). In this study, we applied the mtCOI gene to reconstruct a phylogenetic tree for identifying biotypes B and Q in the Western North Pacific region, and we discuss the relationships between invasive events and ornamental plants. Furthermore, we used genetic analyses of the mtCOI gene to determine the population genetic structure of biotypes $B$ and $Q$ in this region. We attempted to study the genetic diversity within and between populations and determine whether or not biotypes B and Q are invasive agents in this region. We also tried to determine the genetic relationships within and between populations separated by natural barriers. This study provides information for understanding genetic variation of the invasive biotypes of $B$. tabaci in the Western North Pacific region.

\section{Materials and Methods}

Whitefly Samples. Whitefly samples of B. tabaci of the Western North Pacific region were from China,
Japan, Korea, and Taiwan (Table 1; Fig. 1). Specimens of B. tabaci were collected from weeds, vegetables, and ornamental plants (from the wild, farms, and greenhouses, respectively). We selected fourth instars and used classical taxonomic criteria to identify the whitefly species. Adult whiteflies were preserved in $95 \%$ ethanol and stored at $-20^{\circ} \mathrm{C}$. Samples from Cyprus, Israel, the Netherlands, and Spain of the Mediterranean Basin also were analyzed.

COI Gene Sequencing. Genomic DNA was extracted from individual adult whiteflies according to the method suggested by De Barro and Driver (1997). The mitochondrial partial COI gene sequence ( $816 \mathrm{bp}$ ) was amplified by PCR with the primers C1-J-2195 (5'-TTGATTTTTTGGTCATC CAGAAGT-3') and L2-N-3014 (5' -TCCAATGCAC TAATCTGCCATATTA-3') (Frohlich et al. 1999). The PCR reaction program was initialized at $94^{\circ} \mathrm{C}$ for $2 \mathrm{~min}$, followed by 35 cycles of $94^{\circ} \mathrm{C}$ for $1 \mathrm{~min}$, $52^{\circ} \mathrm{C}$ for $1 \mathrm{~min}$, and $72^{\circ} \mathrm{C}$ for $1 \mathrm{~min}$, with a final extension of 5 min at $72^{\circ} \mathrm{C}$. The PCR products were subsequently gel-purified using the Micro-Elute DNA Clean/Extraction Kit (GeneMark, Taipei, Taiwan) and sequenced in one direction on an ABI 3730 DNA Analyzer (Applied Biosystems, Foster, CA) using an ABI PRISM BigDye Terminator Cycle Sequencing Ready Reaction Kit, V3.1 (Applied Biosystems). Sequences obtained in this study were submitted to GenBank, and some samples from the Western North Pacific region in GenBank also were analyzed (Table 1).

Phylogenetic Analysis. Phylogenetic analysis included the B, Q, and other biotypes of B. tabaci ( Tables 1 and 2), with Lipaleyrodes emiliae Chen and Ko (DQ989555) as an outgroup. All sequences were aligned using the Clustal $\times 1.18$ program (Thompson et al. 1997). The resulting alignment was manually edited using the GeneDoc program (Nicholas et al. 1997), and the phylogenetic analysis was performed using Bayesian inference (Yang and Rannala 1997). The best-fitting model of DNA substitution was selected by the Akaike information criterion (AIC) using MrMODELTEST version 2.2 (Nylander 2004). The phylogenetic tree was constructed by using the GTR + G model. The Bayesian analysis was performed using MrBayes version 3.1.2 (Huelsenbeck and Ronquist 2001). Metropolis-coupled Markov chain Monte Carlo analyses were run with four chains (one cold chain and three heated chains). Analyses were initiated with random starting trees and run for $1 \times 10^{6}$ generations and were sampled every 100 generations. For the burn-in period, we discarded 100,000 generations. Posterior clade probabilities were used to assess the levels of nodal support. Branch lengths were saved and are presented on the $50 \%$ majority-rule consensus trees.

Analysis of Genetic Variations and Population Genetics. After our test, we found that samples from the same site revealed the same mtCOI sequences. Therefore, we only selected one individual to represent all specimens from the same site (Table 1; Fig. 1). The mtCOI sequence identities within and among popu- 
Table 1. Samples of biotypes B and Q of B. tabaci from the Western North Pacific region

\begin{tabular}{|c|c|c|c|c|c|c|c|c|}
\hline No. & Acronym & Biotype & Haplotype & $\begin{array}{c}\text { GenBank } \\
\text { accession no. }\end{array}$ & Host plant & Year & Location & Environment \\
\hline 1 & ChinaShanghaiB1 & B & BH6 & AY550274 & Euphorbia pulcherrima & 2003 & China, Shanghai & Field \\
\hline 2 & ChinaNanjingB2 & $\mathrm{B}$ & BH6 & AY518185 & Gossypium hirsutum & 2003 & China, Nanjing & Field \\
\hline 3 & ChinaBeijingB3 & $\mathrm{B}$ & $\mathrm{BH} 6$ & AY582867 & Brassica oleraceae & 2003 & China, Beijing & Field \\
\hline 4 & ChinaHeNanB4 & $\mathrm{B}$ & BH6 & AY582873 & Lycopersicon esculentum & 2003 & China, Henan & Field \\
\hline 5 & ChinaZhejiangB5 & $\mathrm{B}$ & BH6 & AJ867555 & Brassica oleraceae & 2003 & China, Zhejiang & Field \\
\hline 6 & ChinaHainanB6 & $\mathrm{B}$ & $\mathrm{BH} 1$ & AY518187 & Solanum melongena & 2003 & China, Hainan & Field \\
\hline 7 & ChinaXinJiangB7 & $\mathrm{B}$ & $\mathrm{BH} 1$ & AY582868 & Euphorbia pulcherrima & 2003 & China, Xinjiang & Field \\
\hline 8 & ChinaZhejiangB8 & B & BH6 & DQ989520 & Brassica oleraceae & 2005 & China, Zhejiang & Field \\
\hline 9 & ChinaShandongB9 & $\mathrm{B}$ & $\mathrm{BH} 6$ & DQ989521 & Gossypium hirsutum & 2004 & China, Shandong & Field \\
\hline 10 & ChinaShandongB10 & B & BH6 & DQ989522 & Brassica oleraceae & 2004 & China, Shandong & Field \\
\hline 11 & ChinaBeijingB11 & B & BH6 & DQ989523 & Brassica oleracea & 2005 & China, Beijing & Field \\
\hline 12 & ChinaBeijingB12 & $\mathrm{B}$ & $\mathrm{BH} 6$ & DQ989524 & Cucumis sativus & 2005 & China, Beijing & Field \\
\hline 13 & ChinaShanghaiB13 & B & BH6 & DQ989525 & Cucumis sativus & 2005 & China, Shanghai & Field \\
\hline 14 & ChinaGuangdongB14 & $\mathrm{B}$ & BH6 & DQ989526 & Luffa aegyptiaca & 2005 & China, Guangdong & Field \\
\hline 15 & ChinaHubeiB15 & $\mathrm{B}$ & $\mathrm{BH} 6$ & DQ989527 & Brassica oleraceae & 2005 & China, Hubei & Field \\
\hline 16 & ChinaGuangdongB16 & $\mathrm{B}$ & BH6 & DQ989528 & Cucurbita moschata & 2005 & China, Guangdong & Field \\
\hline 17 & ChinaFujianB17 & B & $\mathrm{BH} 6$ & DQ989529 & Brassica oleraceae & 2005 & China, Fujian & Field \\
\hline 18 & ChinaShanxiB18 & $\mathrm{B}$ & $\mathrm{BH} 6$ & DQ989530 & Lycopersicon esculeutum & 2005 & China, Shanxi & Field \\
\hline 19 & KoreaJincheonB1 & B & BH6 & DQ462587 & - & 2006 & Korea, Jincheon & - \\
\hline 20 & KoreaGoyangB2 & B & BH6 & DQ989531 & Rosa hybride & 2003 & Korea, Goyang & Field \\
\hline 21 & KoreaGoyangB3 & $\mathrm{B}$ & $\mathrm{BH} 6$ & DQ174538 & Rosa hybride & 2003 & Korea, Goyang & Field \\
\hline 22 & JapanAINB1 & B & BH6 & AB204577 & Lycopersicon esculeutum & 2004 & Japan, Ehime & Field \\
\hline 23 & JapanKOSB2 & $\mathrm{B}$ & $\mathrm{BH} 4$ & AB204578 & cucurbit plant & 1991 & Japan, Kumamoto & Field \\
\hline 24 & JapanKAKB3 & $\mathrm{B}$ & $\mathrm{BH} 3$ & AB204580 & Lycopersicon esculeutum & 2004 & Japan, Kagoshima & Field \\
\hline 25 & JapanHARB4 & $\mathrm{B}$ & $\mathrm{BH} 2$ & AB204581 & Melon & 2004 & Japan, Kochi & Field \\
\hline 26 & JapanMATB5 & B & $\mathrm{BH} 6$ & AB204582 & Capsicum annuum & 2004 & Japan, Chiba & Field \\
\hline 27 & JapanASAB6 & $\mathrm{B}$ & BH6 & AB204583 & Solanum melongena & 2004 & Japan, Shizuoka & Field \\
\hline 28 & JapanKURB7 & B & BH6 & AB204584 & Lycopersicon esculeutum & 2004 & Japan, Okayama & Field \\
\hline 29 & JapanTOSB8 & $\mathrm{B}$ & BH6 & AB204585 & Melon & 2004 & Japan, Kochi & Field \\
\hline 30 & JapanHonshuB9 & $\mathrm{B}$ & $\mathrm{BH} 6$ & DQ989532 & Euphorbia pulcherrima & 2005 & Japan, Chiba & Field \\
\hline 31 & JapanShikokuB10 & B & BH6 & DQ989533 & Capsicum annuum & 2005 & Japan, Kochi & Field \\
\hline 32 & TaiwanTPB1 & $\mathrm{B}$ & BH6 & DQ989534 & Euphorbia pulcherrima & 2006 & Taiwan, Taipei & Field \\
\hline 33 & TaiwanCYB2 & $\mathrm{B}$ & $\mathrm{BH} 6$ & DQ989535 & Lycopersicon esculeutum & 2006 & Taiwan, Chiayi & Field \\
\hline 34 & TaiwanYLB3 & $\mathrm{B}$ & $\mathrm{BH} 6$ & DQ989536 & Lycopersicon esculeutum & 2006 & Taiwan, Yunlin & Field \\
\hline 35 & TaiwanTNB4 & B & BH6 & DQ989537 & Euphorbia hirta & 2006 & Taiwan, Tainan & Field \\
\hline 36 & TaiwanKHB5 & $\mathrm{B}$ & $\mathrm{BH} 6$ & DQ989538 & Lycopersicon esculeutum & 2006 & Taiwan, Kaohsiung & Field \\
\hline 37 & TaiwanHLB6 & B & BH5 & DQ989539 & Brassica oleraceae & 2006 & Taiwan, Hualien & Field \\
\hline 38 & TaiwanYLB7 & B & BH5 & DQ989540 & Lycopersicon esculeutum & 2006 & Taiwan, Ilan & Field \\
\hline 39 & TaiwanTYB8 & $\mathrm{B}$ & BH6 & DQ989541 & Synedrella nodiflora & 2006 & Taiwan, Taoyuan & Field \\
\hline 40 & TaiwanMLB9 & $\mathrm{B}$ & BH6 & DQ989542 & Euphorbia pulcherrima & 2006 & Taiwan, Miaoli & Greenhouse \\
\hline 41 & TaiwanNTB10 & B & BH6 & DQ989543 & Synedrella nodiflora & 2006 & Taiwan, Nantou & Field \\
\hline 42 & TaiwanCHB11 & $\mathrm{B}$ & $\mathrm{BH} 6$ & DQ989544 & Erigeron bonariensis & 2006 & Taiwan, Changhua & Field \\
\hline 43 & TaiwanTHB12 & $\mathrm{B}$ & BH6 & DQ989545 & Erigeron bonariensis & 2006 & Taiwan, Taichung & Field \\
\hline 44 & ChinaZhejiangQ1 & $\mathrm{Q}$ & $\mathrm{QH} 4$ & DQ473394 & Cucumis sativus & 2006 & China, Zhejiang & Field \\
\hline 45 & ChinaYunNanQ2 & $\mathrm{Q}$ & $\mathrm{QH} 3$ & AY587516 & Euphorbia pulcherrima & 2003 & China, Yunnan & Field \\
\hline 46 & ChinaHeNanQ3 & $\mathrm{Q}$ & $\mathrm{QH} 3$ & AY587514 & Solanum melongena & 2003 & China, Henan & Field \\
\hline 47 & ChinaBeijingQ4 & $\mathrm{Q}$ & $\mathrm{QH} 1$ & AY589499 & Ipomoea nil & 2003 & China, Beijing & Field \\
\hline 48 & ChinaYunnanQ5 & $\mathrm{Q}$ & $\mathrm{QH} 4$ & AY518189 & Euphorbia pulcherrima & 2003 & China, Yunnan & Field \\
\hline 49 & ChinaBeijingQ6 & $\mathrm{Q}$ & $\mathrm{QH} 1$ & AY582872 & Ipomoea nil & 2003 & China, Beijing & Field \\
\hline 50 & KoreaWhaseongQ1 & $\mathrm{Q}$ & $\mathrm{QH} 4$ & DQ462586 & - & 2006 & Korea, Whaseong & - \\
\hline 51 & KoreaGeojeQ2 & $\mathrm{Q}$ & $\mathrm{QH} 3$ & DQ462585 & - & 2006 & Korea, Geoje & - \\
\hline 52 & KoreaJinjuQ3 & $\mathrm{Q}$ & $\mathrm{QH} 3$ & DQ462584 & - & 2006 & Korea, Jinju & - \\
\hline 53 & KoreaBuyeoQ4 & $\mathrm{Q}$ & $\mathrm{QH} 3$ & DQ462583 & - & 2006 & Korea, Buyeo & - \\
\hline 54 & JapanMIHQ1 & $\mathrm{Q}$ & $\mathrm{QH} 3$ & AB204588 & Lycopersicon esculeutum & 2004 & Japan, Hiroshima & Field \\
\hline 55 & JapanOKCQ2 & $\mathrm{Q}$ & $\mathrm{QH} 3$ & AB204587 & Lycopersicon esculeutum & 2004 & Japan, Kagoshima & Field \\
\hline 56 & JapanMYJQ3 & $\mathrm{Q}$ & $\mathrm{QH} 3$ & AB204586 & Melon & 2004 & Japan, Kagoshima & Field \\
\hline 57 & JapanNSGQ4 & $Q$ & $\mathrm{QH} 4$ & AB204579 & Cucurbita maxima & 2004 & Japan, Kumamoto & Field \\
\hline 58 & JapanKyushuQ5 & $Q$ & $\mathrm{QH} 2$ & DQ989546 & Capsicum annuum & 2005 & Japan, Miyazaki & Field \\
\hline 59 & TaiwanTNQ1 & Q & $\mathrm{QH} 3$ & DQ989547 & Euphorbia pulcherrima & 2006 & Taiwan, Tainan, & Potting \\
\hline 60 & TaiwanMLQ2 & $Q$ & QH3 & DQ989548 & Euphorbia pulcherrima & 2006 & Taiwan, Miaoli & Greenhouse \\
\hline 61 & TaiwanMLQ3 & $\mathrm{Q}$ & $\mathrm{QH} 3$ & DQ989549 & Euphorbia pulcherrima & 2006 & Taiwan, Miaoli & Greenhouse \\
\hline 62 & TaiwanMLQ4 & $Q$ & $\mathrm{QH} 3$ & DQ989550 & Euphorbia pulcherrima & 2006 & Taiwan, Miaoli & Greenhouse \\
\hline
\end{tabular}

lations were estimated using GeneDoc (Nicholas et al. 1997). In addition, the genetic distances within and among populations were estimated using Kimura's two-parameter model in MEGA version 3.1 (Kumar et al. 2004). Values of haplotype diversity (h) and nucleotide diversity $(\pi)$ were evaluated with DnaSP version 4.10.8 (Rozas et al. 2003). Analysis of molecular variance (AMOVA) was used to characterize patterns of genetic variation and estimate variance components at different hierarchical levels (among biotypes [Va], among populations within biotypes $[\mathrm{Vb}]$, and within populations $[\mathrm{Vc}]$ ) using Arlequin 


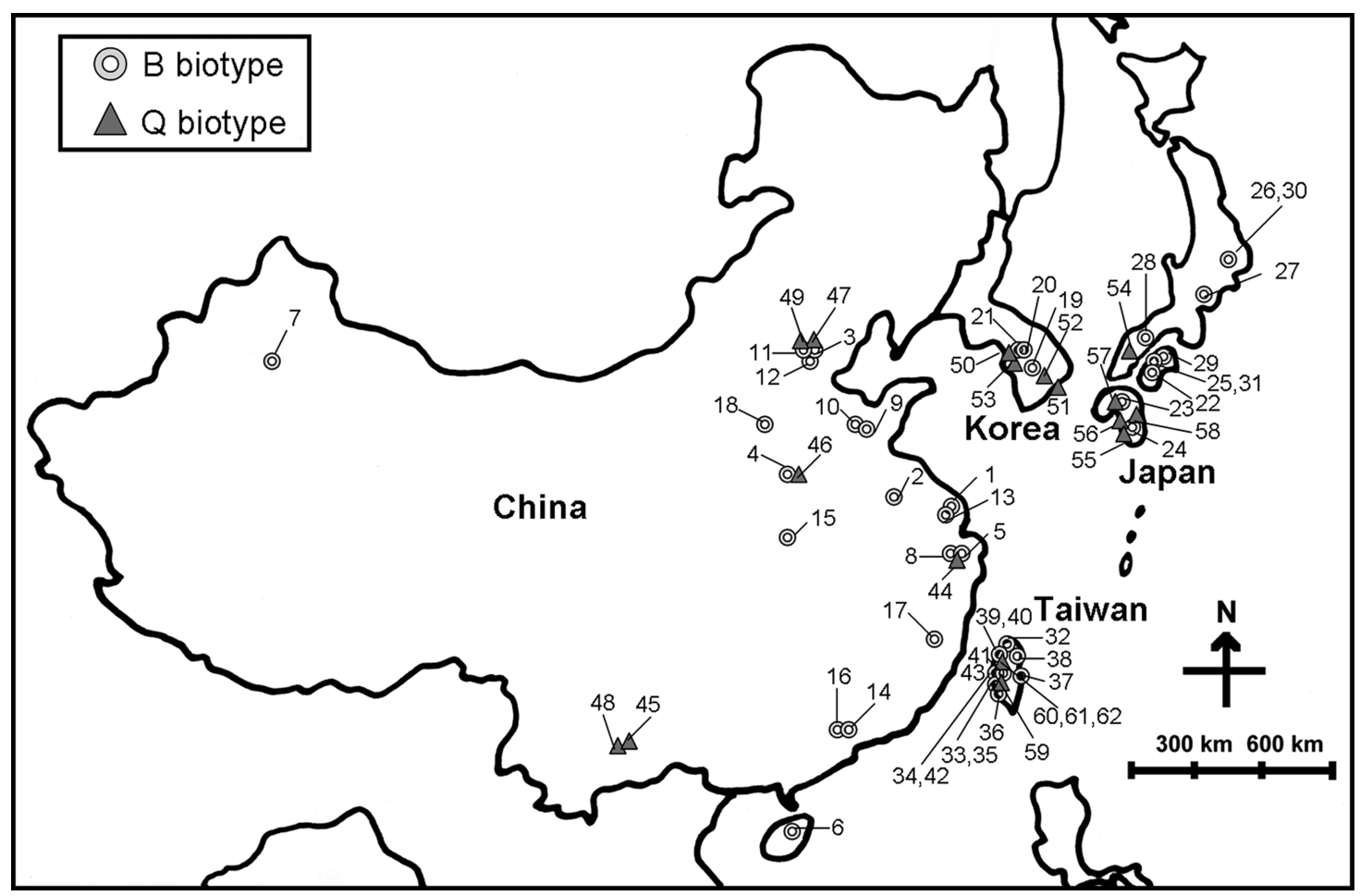

Fig. 1. Locations of samples of B. tabaci biotypes B and Q in the Western North Pacific region. Numbers refer to specimens in Table 1.

version 3.01 (Excoffier et al. 2005). The fixation index of the $\Phi$ statistics was estimated from the pairwise genetic distances among populations (Wright 1951). These distances were also used for the hierarchical analysis of population differentiation (among biotypes $\left[\Phi_{\mathrm{CT}}\right]$, among populations within biotypes $\left[\Phi_{\mathrm{SC}}\right]$, and within populations $\left.\left[\Phi_{\mathrm{ST}}\right]\right)$ (Excoffier et al. 1992). In addition, the statistical significance of the $\Phi$ statistics at different hierarchical levels was tested by 1,000 permutations. The value of $F_{\mathrm{ST}}$ was used to estimate the degree of genetic differentiation among populations (Hudson et al. 1992) using DnaSP 4.10.8.

\section{Results}

Phylogenetic Analysis of B. tabaci Biotypes. Approximately 816 bp of the mtCOI gene was amplified from individual whiteflies using PCR. In addition, we also downloaded sequences from GenBank, and 78 samples were used to reconstruct a phylogenetic tree that included an outgroup (Table 2). After alignment, 441 bp of the mtCOI sequence was used to reconstruct the phylogenetic tree. There were 256 invariable sites, 40 singleton variable sites, and 145 parsimoniously informative sites. The phylogenetic tree based on the Bayesian inference divided B. tabaci into eight biotypes (Fig. 2). Biotypes Q, B, and Ms were clustered into a monophyletic clade with high posterior probability (99\%) support. The results revealed that these three biotypes are sister groups and also indicated that they could are clearly clustered by the three biotypes. Within this group, there was high posterior probability (100\%) support for biotype Ms as a monophyletic group. However, biotypes B and Q clearly belong to different monophyletic subgroups with high posterior probability (99 and $83 \%$, respectively). Therefore, biotypes B and Q are sister groups.

We compared the distributions and host-plant records of biotypes $\mathrm{B}$ and $\mathrm{Q}$ in the Western North Pacific region (Table 1; Fig. 1) (China, Korea, Japan, and Taiwan) relative to the phylogenetic tree (Fig. 2). Biotype Q collected from different host plants of the Western North Pacific region were clustered in the same clade which also included samples from the Mediterranean Basin. Samples of biotype B from the Western North Pacific region clustered in the same clade as a polytomy, along with samples from the Mediterranean Basin and around the world. The branch lengths of samples of biotype B were similar, revealing that their relationships were close. Biotype $\mathrm{Q}$ branch lengths also were similar, indicating close relationships of samples from the Western North Pacific region.

Genetic Variation and Population Genetics of Biotypes $B$ and $Q$ in the Western North Pacific Region. After sequence alignment, $473 \mathrm{bp}$ of the mtCOI sequence was used to analyze genetic variation and population genetics of biotypes B and Q in the Western North Pacific region. Comparison of 43 samples of biotype $\mathrm{B}$ from this region revealed 468 sequence- 
Table 2. Samples of B. tabaci whose mtCOI sequences were used to construct the phylogenetic tree in Fig. 2

\begin{tabular}{|c|c|c|c|c|c|}
\hline Acronym & Biotype & $\begin{array}{c}\text { GenBank } \\
\text { accession no. }\end{array}$ & Host plant & Year & Location \\
\hline AusBF5 & B & DQ174535 & - & - & Australia \\
\hline ArizonaB & B & AY057123 & Euphorbia pulcherrima & 2001 & USA, Arizona \\
\hline SouthAfricaB & $\mathrm{B}$ & AY057140 & Lycopersicon esculeutum & 2001 & South Africa \\
\hline FranceB & B & AJ550170 & Solanum melongena & 2003 & France, Antibes \\
\hline IndiaB & $\mathrm{B}$ & AF321927 & Lycopersicon esculeutum & 2000 & India, Kolar \\
\hline ArgentinaB & B & AF340215 & - & 2001 & Argentina, Buenos Aires \\
\hline ReunionB & $\mathrm{B}$ & AJ550177 & Solanum melongena & 2003 & Reunion \\
\hline UgandaB & B & AY903569 & Hibiscus esculentus & 2005 & Uganda, Namulonge \\
\hline SpainF32B & $\mathrm{B}$ & DQ989551 & Lycopersicon esculentum & 2003 & Spain \\
\hline IsraelF60B & B & DQ989552 & - & 2006 & Israel \\
\hline TurkeyQ & $\mathrm{Q}$ & AF342776 & Gossypium arboreum & 2001 & Turkey \\
\hline FranceQ & $\mathrm{Q}$ & AM180063 & - & 2005 & France, Roussillon \\
\hline AlgeriaQ & $\mathrm{Q}$ & AM176575 & Cucurbita sp. & 2005 & Algeria, Biskra \\
\hline MoroccoQ & $\mathrm{Q}$ & AM176573 & Lycopersicon esculentum & 2005 & Morocco, Biougra \\
\hline TurkeyQ & $\mathrm{Q}$ & AF342776 & Gossypium arboreum & 2001 & Turkey \\
\hline Cameroon $\mathrm{Q}$ & $\mathrm{Q}$ & AF344258 & Hibiscus esculentus & 2001 & Cameroon, Banga-Bakundu \\
\hline ZimbabweQ & $\mathrm{Q}$ & AF344285 & - & 2001 & Zimbabwe, Mazowe \\
\hline SudanQ & $\mathrm{Q}$ & AY827613 & Gossypium arboreum & 2004 & Sudan, \\
\hline NetherlandsQF13 & $\mathrm{Q}$ & DQ174541 & Hibiscus sp. & 2002 & Netherlands \\
\hline SpainQF7 & $\mathrm{Q}$ & DQ174539 & - & 2002 & Spain \\
\hline CyprusF42Q & $\mathrm{Q}$ & DQ989553 & - & 2004 & Cyprus \\
\hline IsraelF61Q & $\mathrm{Q}$ & DQ9895554 & - & 2006 & Israel \\
\hline ArgentinaA & A & AF340212 & Gossypium arboreum & 2001 & Argentina, Santiago \\
\hline ArizonaA & A & AY057122 & Gossypium arboreum & 1983 & Arizona \\
\hline BoliviaA & A & AF342768 & Lycopersicon esculeutum & 1999 & Bolivia \\
\hline MexicoA & A & AY057125 & Lycopersicon esculeutum & 1989 & Mexico \\
\hline ColombiaA & A & AJ550168 & Chromolaena odorata & 2003 & Colombia, Cali \\
\hline HondurasA & A & AF342770 & Melon & 1998 & Honduras \\
\hline TanzaniaC & Cassava & AF418667 & Manihot esculenta & 2004 & Tanzania, Mtwara \\
\hline GhanaC & Cassava & AF418668 & Manihot esculenta & 2004 & Ghana, Accra \\
\hline MalawiC & Cassava & AY057215 & Manihot esculenta & 2001 & Malawi \\
\hline UgandaBMC & Cassava & AY057142 & Manihot esculenta & 1997 & Uganda \\
\hline UgandaBC & Cassava & AY057141 & Manihot esculenta & 1997 & Uganda \\
\hline ZambiaC & Cassava & AF344281 & - & 2001 & Zambia \\
\hline SwazilandC & Cassava & AF344269 & - & 2001 & Swaziland \\
\hline SouthAfricaC & Cassava & AF344260 & - & 2001 & South Africa \\
\hline MozambiqueC & Cassava & AF344278 & - & 2001 & Mozambique \\
\hline MadagascarMs & Ms & AJ550171 & Lycopersicon esculentum & 2003 & Madagascar, Tulear \\
\hline MauritiusMs & Ms & AJ550172 & Phaseolus sp. & 2003 & Mauritius \\
\hline SeychellesMs & Ms & AJ550182 & Jatropha sp. & 2003 & Seychelles, Mahe \\
\hline ReunionMs & Ms & AJ550178 & Euphorbia pulcherrima & 2003 & Reunion \\
\hline UgandaMs & Ms & AY903524 & Bean & 2005 & Uganda \\
\hline ItalyPT1 & $\mathrm{T}$ & AY 827595 & Euphorbia characias & 2004 & Italy, Puglia \\
\hline ItalyPT2 & $\mathrm{T}$ & AY827597 & Euphorbia characias & 2004 & Italy, Puglia \\
\hline ItalyST1 & $\mathrm{T}$ & AY 827600 & Euphorbia characias & 2004 & Italy, Sicily \\
\hline ItalyST2 & $\mathrm{T}$ & AY827601 & Euphorbia characias & 2004 & Italy \\
\hline ItalyST3 & $\mathrm{T}$ & AY827602 & Euphorbia characias & 2004 & Italy \\
\hline ItalyST4 & $\mathrm{T}$ & AY827603 & Euphorbia characias & 2004 & Italy \\
\hline India2 & Nauru & AJ748374 & Solenidiopsis peruvianum & 2004 & India, Karnataka \\
\hline Nepal & Nauau & AF342779 & Citrullus lanatus & 2001 & Nepal \\
\hline Pakistan2 & Nauau & AY057582 & Gossypium arboreum & 2001 & Pakistan \\
\hline TaiwanNaurul & Nauru & DQ174518 & Boehmeria nivea & 2003 & Taiwan, Hsinchu \\
\hline TaiwanNauru2 & Nauru & DQ174519 & Humulus scandens & 2002 & Taiwan, Chiayi \\
\hline TaiwanNauru3 & Nauru & DQ174520 & Ipomoea acuminata & 2003 & Taiwan, Taitung, \\
\hline TaiwanNauru4 & Nauru & DQ174521 & Euphorbia pulcherrima & 2002 & Taiwan, Kaohsiung \\
\hline ChinaNaurul & Nauru & DQ174522 & Gossypium hirsutum & 2004 & China, Jiangsu \\
\hline ChinaNauru2 & Nauru & DQ174523 & Codiaeum variegatum & 2004 & China, Guangdong \\
\hline Indonesia & Nauru & DQ174524 & Capsicam annunm & 2003 & Indonesia \\
\hline AustraliaAn & An & DQ174529 & - & - & Australia \\
\hline TaiwanAnl & An & DQ174525 & Momordica ochinchinensis & 2002 & Taiwan, Chiayi \\
\hline TaiwanAn2 & An & DQ174526 & Achyranthes obtusifolia & 2003 & Taiwan, Kaohsiung \\
\hline TaiwanAn3 & An & DQ174527 & Mesona chinensis & 2003 & Taiwan, Hualien \\
\hline TaiwanAn4 & An & DQ174528 & Solanaceae & 2003 & Taiwan, Hsinchu \\
\hline ChinaAn & An & AF342777 & Gossypium arboreum & 2001 & China \\
\hline Malaysia & An & AY057137 & - & 2001 & Malaysia \\
\hline Indial & An & AJ748365 & Field bean & 2004 & India, Karnataka \\
\hline India3 & An & AJ748360 & Solanum melongena & 2004 & India, Karnataka \\
\hline Pakistan1 & An & AF164669 & Cucurbita moschata & 1999 & Pakistan \\
\hline Thailand & An & AF164670 & Gossypium arboreum & 1999 & Thailand \\
\hline
\end{tabular}




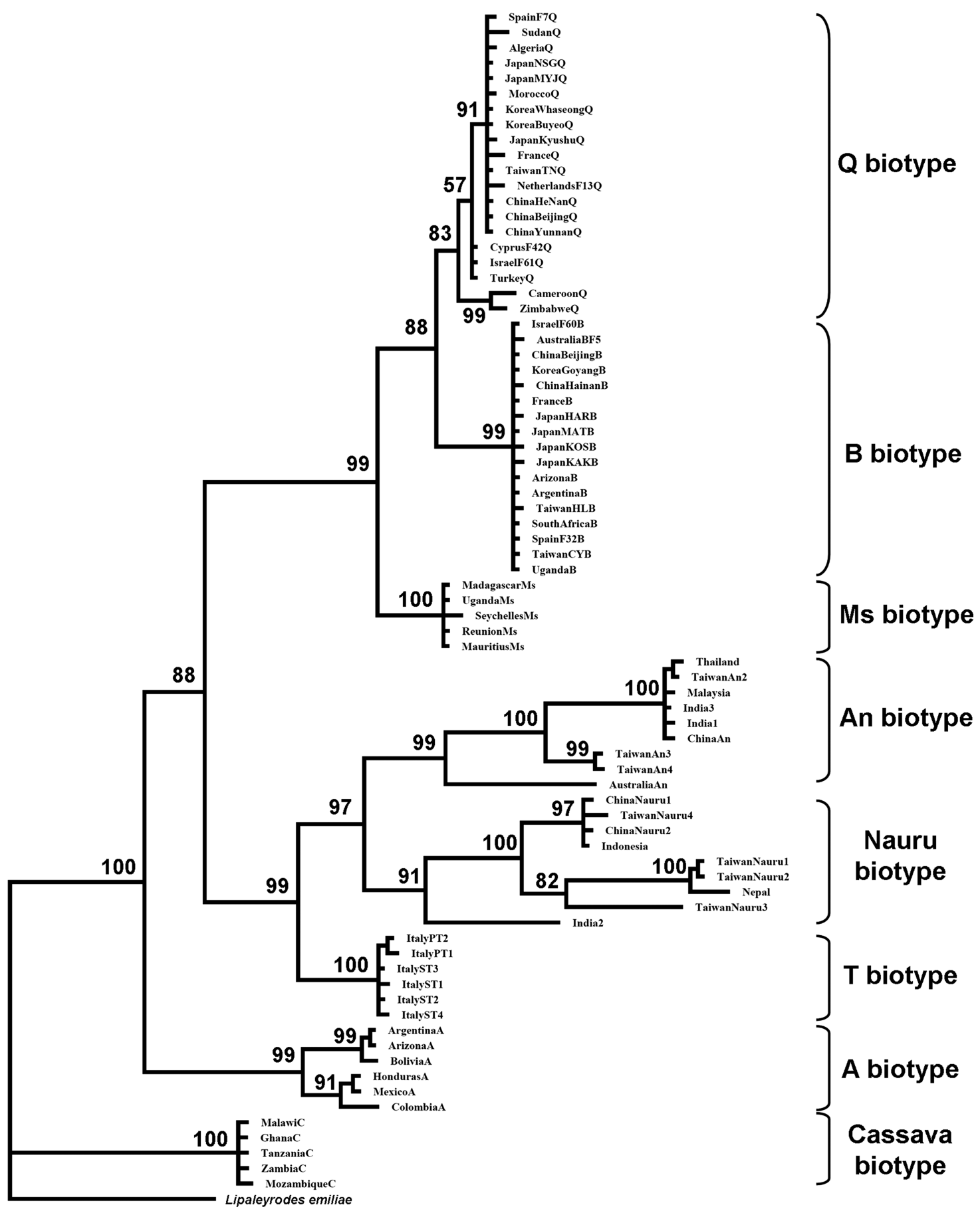

- 0.1 Substitutions/site

Fig. 2. Phylogenetic tree of mtCOI sequences for B. tabaci based on Bayesian inferences. Numbers at the nodes are the posterior probabilities as support values. The outgroup was L. emiliae.

invariable sites, 3 singleton-variable sites, and 2 parsimoniously informative sites. Therefore, overall sequences of these samples revealed six haplotypes in this region (Table 3), and $\mathrm{BH} 6$ was the most common haplotype in all four populations. Furthermore, BH1 was found only in China, BH5 was found only in Taiwan, and BH2 and BH3 were found only in Japan. The highest haplotype $(\mathrm{h}=0.53333)$ and nucleotide 
Table 3. Distribution of haplotypes, haplotype diversity $(h)$, and nucleotide diversity $(\pi)$ based on mtCOI sequences within populations of B. tabaci $B$ and $Q$ biotypes from Western North Pacific region

\begin{tabular}{|c|c|c|c|c|c|c|c|c|c|}
\hline \multirow{2}{*}{ Biotype } & \multirow{2}{*}{$\begin{array}{l}\text { Population } \\
(n)\end{array}$} & \multicolumn{6}{|c|}{ Haplotype } & \multirow{2}{*}{$\begin{array}{l}\text { Haplotype } \\
\text { diversity (h) }\end{array}$} & \multirow{2}{*}{$\begin{array}{l}\text { Nucleotide } \\
\text { diversity }(\pi)\end{array}$} \\
\hline & & $\mathrm{BH} 1$ & $\mathrm{BH} 2$ & $\mathrm{BH} 3$ & $\mathrm{BH} 4$ & BH5 & BH6 & & \\
\hline \multirow[t]{5}{*}{ B } & ChinaB (18) & 2 & & & & & 16 & 0.20915 & 0.00044 \\
\hline & KoreaB (3) & & & & & & 3 & - & - \\
\hline & JapanB (10) & & 1 & 1 & 1 & & 7 & 0.53333 & 0.00127 \\
\hline & TaiwanB (12) & & & & & 2 & 10 & 0.30303 & 0.00064 \\
\hline & Total & 2 & 1 & 1 & 1 & 2 & 36 & 0.30011 & 0.00068 \\
\hline \multirow[t]{6}{*}{ Q } & & QH1 & $\mathrm{QH} 2$ & QH3 & QH4 & & & & \\
\hline & ChinaQ (6) & 2 & & 2 & 2 & & & 0.8 & 0.00226 \\
\hline & KoreaQ (4) & & & 3 & 1 & & & 0.5 & 0.00106 \\
\hline & JapanQ (5) & & 1 & 3 & 1 & & & 0.7 & 0.00169 \\
\hline & TaiwanQ (4) & & & 4 & & & & - & - \\
\hline & Total & 2 & 1 & 12 & 4 & & & 0.57310 & 0.00138 \\
\hline
\end{tabular}

diversities $(\pi=0.00127)$ were found among individuals of the Japanese population. In addition, the lowest haplotype $(\mathrm{h}=0.20915)$ and nucleotide diversities ( $\pi=0.00044)$ were found among individuals of the Chinese population. Across all samples of this region, haplotype diversity (h) was 0.30011 and nucleotide diversity $(\pi)$ was 0.00068 . However, we could not calculate the haplotype or nucleotide diversity for the Korean population because there was only one haplotype.

A comparison of 19 samples of biotype $Q$ from the Western North Pacific region revealed 470 sequenceinvariable sites, 1 singleton-variable site, and 2 parsimoniously informative sites. There were four haplotypes in this region (Table 3), with QH3 being the most common. The highest haplotype $(\mathrm{h}=0.8)$ and nucleotide diversities $(\pi=0.00226)$ were found among individuals of the Chinese population. The lowest haplotype $(\mathrm{h}=0.5)$ and nucleotide diversities ( $\pi=0.00106$ ) were found among individuals of the Korean population. Across all samples of this region, haplotype diversity (h) was 0.57310 and nucleotide diversity $(\pi)$ was 0.00138 . We could not calculate the haplotype or nucleotide diversity for the Taiwanese population because there was only one haplotype.

Hierarchical analysis by AMOVA detected that the great majority of variation was among biotypes B and Q (98\%; Table 4). The variation among populations within biotypes was only $0.05 \%$. In addition, the variation within populations $(1.94 \%)$ was higher than that among populations within biotypes. Population differentiation of the $\Phi$ statistics accounted for three sources of variation (Table 4 ). A significant $\Phi_{\mathrm{ST}}$ value was detected within populations $\left(\Phi_{\mathrm{ST}}=0.98056, P<\right.$ 0.001). A significant $\Phi_{\mathrm{CT}}$ value was detected among biotypes $\left(\Phi_{\mathrm{CT}}=0.98004, P<0.05\right)$. The results indicated that genetic differences among biotypes were responsible for the differences within populations. No significant $\Phi_{\mathrm{SC}}$ value was observed among populations within biotypes $\left(\Phi_{\mathrm{SC}}=0.02565\right)$.

Sequence identity and genetic distance (of Kimura's two-parameter model) were used to compare populations. Sequence identities between populations of biotype B in the Western North Pacific region were all 99-100\%, and those within populations were also $99-$ $100 \%$. The average genetic distance between populations was $0.00024-0.00099$. The maximum distance was 0.00099 between the Japanese and Taiwanese populations. The minimum distance was 0.00024 between the Chinese and Korean populations. The mean distance within populations was $0-0.00127$. Sequences from different individuals within the Korean population of biotype B were identical. Sequence identities among populations of biotype $\mathrm{Q}$ in the Western North Pacific region were $99-100 \%$, whereas those within populations were $99-100 \%$. Mean genetic distances between populations were 0.00053-0.00198. Average distances within populations were 0-0.00226. Sequences from different individuals within the Taiwanese population of biotype $Q$ were identical.

Genetic differentiation between populations was estimated by pairwise $F_{\mathrm{ST}}$ (Table 5). Comparisons among populations of biotype B in the Western North Pacific region resulted in low $F_{\mathrm{ST}}$ values, suggesting no genetic differentiation among populations of biotype $\mathrm{B}$ in this region. Likewise, pairwise comparisons among populations of biotype $\mathrm{Q}$ in the Western North Pacific region also resulted in low $F_{\mathrm{ST}}$ values, indicating no genetic differentiation among populations of biotype $\mathrm{Q}$ in this region.

Table 4. Hierarchical analysis of molecular variance and $\Phi$ statistics of genetic differences for $B$. tabaci biotypes $B$ and $Q$ from the Western North Pacific region

\begin{tabular}{lrccc}
\hline \hline \multicolumn{1}{c}{ Source of variation } & df & Sum of squares & Variance components & Percent of variation \\
\hline Among biotypes & 1 & 274.797 & $10.41741 \mathrm{Va}$ & 98 \\
Among populations within biotypes & 6 & 1.478 & $0.00544 \mathrm{Vb}$ & $\Phi_{\mathrm{CT}}: 0.98004^{a}$ \\
Within populations & 54 & 11.161 & $0.20669 \mathrm{Vc}$ & 0.05 \\
Total & 61 & 287.435 & 10.62953 & 1.94 \\
\hline
\end{tabular}

Significant population structure is tested among biotypes, among populations within biotypes, and within populations.

${ }^{a} p<0.05 ;{ }^{b} p<0.001$. 
Table 5. Pairwise estimates of $\boldsymbol{F}_{\mathrm{ST}}$ among populations of $\boldsymbol{B}$. tabaci biotypes $B$ and $Q$ from the Western North Pacific region

\begin{tabular}{llccc}
\hline \hline & & 1 & 2 & 3 \\
\hline B biotype $(n)$ & 1 ChinaB (18) & & & \\
& 2 KoreaB (3) & 0.05882 & & \\
& 3 JapanB (10) & 0.0159 & $<0.00001$ & \\
& 4 TaiwanB (12) & 0.07807 & 0.09091 & 0.03247 \\
Q biotype $(n)$ & & 1 & 2 & 3 \\
& 1 ChinaQ (6) & & & \\
& 2 KoreaQ (4) & -0.04444 & & \\
& 3 JapanQ (5) & $<0.00001$ & -0.18182 & \\
& 4 TaiwanQ (4) & 0.2 & $<0.00001$ & $<0.00001$ \\
\hline
\end{tabular}

\section{Discussion}

The phylogenetic tree based on Bayesian inferences revealed that biotypes $\mathrm{B}$ and $\mathrm{Q}$ of $\mathrm{B}$. tabaci are both present in China, Korea, Japan, and Taiwan of the Western North Pacific region. Biotype B has already been reported from these four countries, but biotype $Q$ was known only from China until a recent report from Japan (Zhang et al. 2005, Hsieh et al. 2006, Ueda and Brown 2006). Biotype $Q$ was detected for the first time in Taiwan in this study. Biotype B is an invader worldwide and biotype $\mathrm{Q}$ is a recent invader of China, Japan, and the United States (Perring 2001, Zhang et al. 2005, Ueda and Brown 2006). Therefore, we hypothesize that biotype $Q$ is a new invader of Taiwan.

Results of the phylogenetic tree provide some information on the invasive events of biotypes $B$ and $Q$ in the Western North Pacific region. Biotype B of this region clustered with the worldwide samples, forming a clade that was a polytomy. This result supports previous studies that found that biotype $\mathrm{B}$ has been spread rapidly across the globe by human trade activities (Frohlich et al. 1999, De Barro et al. 2000, Perring 2001). Therefore, biotype $B$ in this region undoubtedly represents an invasive event, as suggested in previous reports (Zhang et al. 2005, Hsieh et al. 2006, Ueda and Brown 2006). The biotype $Q$ clade was divided into three branches and appeared more genetically polymorphic. The tree topology revealed that biotype Q samples from this region clustered with Mediterranean countries. Although biotype $\mathrm{Q}$ was thought to be restricted originally to the Iberian Peninsula, it has recently been found in various countries around the Mediterranean basin (Brown et al. 2000, Horowitz et al. 2005). Consequently, we hypothesize that the invasion by biotype $Q$ in the Western North Pacific region originated recently from one of several possible Mediterranean countries.

The routes of invasive biotypes of $B$. tabaci are believed to be related to the international trade in ornamentals (Brown et al. 1995, De Barro 1995). Imported poinsettias were probably the source of biotype $\mathrm{B}$, which dispersed from ornamental greenhouses into the field in the United States (Brown et al. 1995). Invasions of biotype $\mathrm{Q}$ were hypothesized to be associated with ornamental crops in China and Japan (Zhang et al. 2005, Ueda and Brown 2006). Fortuitously, we found direct evidence to support this hy- pothesis. There was no evidence of biotype $\mathrm{Q}$ in Taiwan before 2005 (Hsieh et al. 2006); in 2006 we recorded biotype $\mathrm{Q}$ only in one greenhouse containing poinsettias. The poinsettia plantlets had been obtained from Italy in 2006. Furthermore, we did not find any biotype $\mathrm{Q}$ whiteflies outside the greenhouse or in the field in Taiwan in 2006. Thus, we consider this to be powerful evidence supporting the hypothesis that routes of invasion by biotypes of $B$. tabaci are related to the international ornamentals trade.

Population genetic analyses were used to study genetic variation of invasive biotypes and to reconstruct the invasive events. According to haplotype analyses, BH6 was the only haplotype shared among countries, whereas the other five haplotypes were unique to a given country. In addition, haplotype and nucleotide diversities within each country were very low. Six haplotypes with low levels of genetic variability were observed, which suggests that multiple invasive events or rapid divergence have occurred. However, the molecular clock hypothesis based on Brower's estimates for mitochondrial DNA indicated 2.3\% pairwise sequence divergence per million years (Brower 1994). Therefore, we hypothesize that the invasive biotype $\mathrm{B}$ in this region exists because of multiple invasive events. However, QH3 was found to be the most common haplotype within each country. Haplotype QH4 also was shared among countries besides Taiwan, while the other two haplotypes were unique to individual countries. Haplotype and nucleotide diversities also revealed low genetic variability within each country. Based on our results, we hypothesize that the invasive event of biotype $Q$ in Taiwan was a single event, because only the QH4 haplotype was found in one poinsettia greenhouse. Although the ocean provides a natural barrier between China and Japan and between Korea and Japan, they all share two haplotypes. This suggests that the invasion of biotype $Q$ in this region may have occurred through multiple invasive events. A recently introduced population that has expanded in size from a low number of founders is expected to have a common haplotype which is shared by a majority of individuals and many rarer haplotypes with a few independent mutations (Avise 2000). Based on the genetic variability of the population genetics analyses, we hypothesize that the invasions of biotypes $B$ and $Q$ in the Western North Pacific region occurred through multiple invasive events.

Hierarchical analysis with AMOVA was used to characterize patterns of genetic variations and to estimate variance components. The results revealed that the great majority of variation was among biotypes B and $Q$, whereas there were also significant genetic differences among $\mathrm{B}$ and $\mathrm{Q}$ biotypes. This supports biotypes B and Q being genetically different, as previously reported (Moya et al. 2001). The fixation index of the $\Phi$ statistics was used for the hierarchical analysis of population differentiation. We noted that there was relatively large differentiation among biotypes, which was responsible for differences within populations. The results revealed higher levels of genetic differ- 
ences within populations than among populations as a whole, a result that is unreasonable given the ocean as a natural barrier. Thus, the combined results of AMOVA analyses supported the hypothesis of multiple invasive events of biotypes B and Q in the Western North Pacific region.

The population genetic structure was studied to determine the genetic relationships within populations and between populations of invasive biotypes of B. tabaci in this region. Similar results of low genetic distances and high sequence identities between and within populations of biotype $B$ revealed no differences between populations. Low $F_{\text {ST }}$ values between populations of biotype $\mathrm{B}$ also revealed low levels of genetic differentiation. These results indicate that few differences exist among populations. However, it seems impossible that no differences would exist between long-resident natural populations with the ocean serving as a natural barrier. Instead, one would expect to observe isolation by distance, where genetic similarity among populations decreases as the geographic distance between them increases (Jensen et al. 2005). We found that biotypes B and Q in this region do not exhibit. Consequently, the population structure analyses support hypothesis of recent multiple invasions of biotypes $\mathrm{B}$ and $\mathrm{Q}$ in this region.

Actually, biotypes B and Q are known invaders of the Western North Pacific region. Biotype B has already become an important pest and has transmitted begomoviruses that have caused economic losses in this region (Zhang et al. 2005, Hsieh et al. 2006, Ueda and Brown 2006). Therefore, biotype B has already passed through the three stages of invasion-i.e., importation, introduction, and establishment (Williamson and Fitter 1996) — to become a pest species. Furthermore, biotype Q in China, Korea, and Japan has also passed through the three stages to become a pest. However, we found biotype $Q$ in Taiwan only in a poinsettia greenhouse, revealing that it is only in the importation stage, and has not yet been introduced (or released) to the environment.

Whether biotype B of B. tabaci is an invader has long been controversial. Molecular markers and viral disease are used to show incursions of biotype $\mathrm{B}$ and have indicated that it was transported on ornamental plants (Brown et al. 1995, Frohlich et al. 1999, De Barro et al. 2000). Furthermore, this study supports the invasion of biotype $\mathrm{Q}$ being a recent event related to ornamental plants, and it can be expected that it will soon spread worldwide as did biotype B through human trade activities. However, inherent levels of resistance to insecticides differ in biotypes B and Q (Khasdan et al. 2005). Insecticide applications have affected the dynamics and distribution of biotypes B and Q elsewhere (Khasdan et al. 2005). Therefore, ornamental crops should be quarantined to prevent the future invasion of these biotypes, and better ways to eliminate and control these two pests should be sought. Molecular markers will play an important role in monitoring and preventing their further invasion.

\section{Acknowledgments}

We thank A. R. Horowitz (Department of Entomology, Agricultural Research Organization, Gilat Research Center, Israel) for supplying the Israel samples, I. Bedford (John Innes Centre, Norwich, UK) for supplying the Spain samples, M. Jansen (Plant Protection Service, Wageningen, the Netherlands) for supplying the Netherlands samples, S. J. Suh (National Plant Quarantine Service, Goyang, South Korea) for supplying the Korea samples, K. I. Honda and S. Ueda (National Institute of Vegetable and Tea Science, Japan) for supplying the Japan samples, and C. Luo and X. J. Guo (Institute of Plant and Environment Protection, Beijing Academy of Agriculture and Forestry Science, China), B. L. Qiu (Entomology Department, South China Agricultural University, Guangzhou, China), and S. S. Liu (Institute of Applied Entomology, Zhejiang University, China) for supplying the China samples. We thank Y. C. Hung, C. H. Chen, Y. F. Chen, F. S. Wu, Y. T. Shih, and H. T. Yeh (National Taiwan University (NTU), Taipei, Taiwan) for help with sample collection, and S. K. Green (The Asian Vegetable Research and Development Center, Tainan, Taiwan) for providing samples. We thank C. F. Hsieh and S. C. Wu (Department of Life Science, NTU) for identifying host plants of B. tabaci; X. Y. Tsai (NTU), H. F. Hung (NTU), Y. H. Chen, and Y. C. Lo for assistance; and D. Chamberlin for editing the English of the draft. This paper was supported, in part, by grants from the National Science Council (NSC952621-B-002-0120) and Bureau of Animal and Plant Health Inspection and Quarantine (94 AS-13.3.1-BQ-B2), Taiwan.

\section{References Cited}

Abdullahi, I., S. Winter, G. I. Atiri, and G. Thottappilly. 2003. Molecular characterization of whitefly, Bemisia tabaci (Hemiptera: Aleyrodidae) populations infesting cassava. Bull. Entomol. Res. 93: 97-106.

Avise, J. C. 2000. Phylogeography: the history and formation of species. Harvard University Press, Cambridge, MA.

Berry, S. D., V. N. Fondong, C. Rey, D. Rogan, C. M. Fauquet, and J. K. Brown. 2004. Molecular evidence for five distinct Bemisia tabaci (Homoptera: Aleyrodidae) geographic haplotypes associated with cassava plants in subSaharan Africa. Ann. Entomol. Soc. Am. 97: 852-859.

Brower, A.V.Z. 1994. Rapid morphological radiation and convergence among races of the butterfly Heliconius erato inferred from patterns of mitochondrial DNA evolution. Proc. Natl. Acad. Sci. U.S.A. 91: 6491-6495.

Brown, J. K., D. R. Frohlich, and R. C. Rosell. 1995. The sweetpotato or silverleaf whiteflies: biotypes of Bemisia tabaci or a species complex? Annu. Rev. Entomol. 40: 511-534.

Brown, J. K., T. M. Perring, A. D. Cooper, I. D. Bedford, and P. G. Markham. 2000. Genetic analysis of Bemisia (Hemiptera: Aleyrodidae) populations by isoelectric focusing electrophoresis. Biochem. Genet. 38: 13-25.

De Barro, P. J. 1995. Bemisia tabaci biotype B: a review of its biology, distribution and control. CSIRO, Canberra, Australia.

De Barro, P. J. 2005. Genetic structure of whitefly Bemisia tabaci in the Asia-Pacific region revealed using microsatellite markers. Mol. Ecol. 14: 3695-3718.

De Barro, P. J., and F. Driver. 1997. Use of RAPD to distinguish the B biotype from other biotypes of Bemisia tabaci (Hemiptera: Aleyrodidae). Aust. J. Entomol. 36: $149-152$.

De Barro, P. J., F. Driver, J.W.H. Trueman, and J. Curran. 2000. Phylogenetic relationships of world populations of 
Bemisia tabaci (Gennadius) using ribosomal ITS1. Mol. Phylogenet. Evol. 16: 29-36.

De la Rúa, P., B. Simón, D. Cifuentes, C. Martinez-Mora, and J. L. Cenis. 2006. New insights into the mitochondrial phylogeny of the whitefly Bemisia tabaci (Hemiptera: Aleyrodidae) in the Mediterranean Basin. J. Zool. Syst. Evol. Res. 44: 25-33.

Excoffier, L., G. Laval, and S. Schneider. 2005. Arlequin ver. 3.0: an integrated software package for population genetics data analysis. Evol. Bioinform. Online 1: 47-50.

Excoffier, L., P. E. Smouse, and J. M. Quattro. 1992. Analysis of molecular variance inferred from metric distances among DNA haplotypes: application to human mitochondrial DNA restriction data. Genetics 131: 479-491.

Frohlich, D. R., I. Torres-Jerez, I. D. Bedford, P. G. Markham, and J. K. Brown. 1999. A phylogeographical analysis of the Bemisia tabaci species complex based on mitochondrial DNA markers. Mol. Ecol. 8: 1683-1691.

Harrison, R. G. 1989. Animal mitochondrial DNA as a genetic marker in population and evolutionary biology. Trends Ecol. Evol 4: 6-11.

Horowitz, A. R., S. Kontsedalov, V. Khasdan, and I. Ishaaya. 2005. Biotypes B and Q of Bemisia tabaci and their relevance to neonicotinoid and pyriproxyfen resistance. Arch. Insect Biochem. Physiol. 58: 216-225.

Hsieh, C. H., C. H. Wang, and C. C. Ko. 2006. Analysis of Bemisia tabaci (Hemiptera: Aleyrodidae) species complex and distribution in eastern Asia based on mitochondrial DNA markers. Ann. Entomol. Soc. Am. 99: 768-775.

Hudson, R. R., M. Slatkin, and W. P. Maddison. 1992. Estimation of levels of gene flow from DNA sequence data. Genetics 132: 583-589.

Huelsenbeck, J. P., and F. Ronquist. 2001. MrBayes: Bayesian inference of phylogenetic trees. Bioinformatics 17: $754-755$.

Jensen, J. L., A. J. Bohonak, and S. T. Kelley. 2005. Isolation by distance, web service. BMC Genet. 6: 13-18.

Khasdan, V., I. Levin, A. Rosner, S. Morin, S. Kontsedalov, L. Maslenin, and A. R. Horowitz. 2005. DNA markers for identifying biotypes B and Q of Bemisia tabaci (Hemiptera: Aleyrodidae) and studying population dynamics. Bull. Entomol. Res. 95: 605-613.
Kumar, S., S. Tamura, and M. Nei. 2004. MEGA3: integrated software for molecular evolutionary genetics analysis and sequence alignment. Brief Bioinform. 5: 150-163.

Moya, A., P. Guirao, D. Cifuentes, F. Beitia, and J. L. Cenis. 2001. Genetic diversity of Iberian populations of Bemisia tabaci (Hemiptera: Aleyrodidae) based on random amplified polymorphic DNA-polymerase chain reaction. Mol. Ecol. 10: 891-897.

Nicholas, K. B., H. B. Nicholas, Jr., and D. W. Deerfield, II. 1997. GeneDoc: analysis and visualization of genetic variation. Embnew News. 4: 14.

Nylander, J.A.A. 2004. MrModeltest v2. Program distributed by the author. Evolution Biology Centre, Uppsala University, Uppsala, Sweden.

Perring, T. M. 2001. The Bemisia tabaci species complex. Crop Protect. 20: 725-737.

Rozas, J., J. C. Sánchez-DelBarrio, X. Messeguer, and R. Rozas. 2003. DnaSP, DNA polymorphism analyses by the coalescent and other methods. Bioinformatics 19: 2496-2497.

Thompson, J. D., T. J. Gibson, F. Plewniak, F. Jeanmougin, and D. G. Higgins. 1997. The ClustalX windows interface: flexible strategies for multiple sequence alignment aided by quality analysis tools. Nucleic Acids Res. 24: 4876-4882.

Ueda, S., and J. K. Brown. 2006. First report of Q biotype of Bemisia tabaci in Japan by mitochondrial cytochrome oxidase I sequence analysis. Phytoparasitica 34: 405-411.

Williamson, M. H., and A. Fitter. 1996. The characters of successful invaders. Biol. Conserv. 78: 163-170.

Wright, S. 1951. The genetical structure of populations. Ann. Eugenics 15: 323-354.

Yang, Z., and B. Rannala. 1997. Bayesian phylogenetic inference using DNA sequences: a Markov chain Monte Carlo method. Mol. Biol. Evol. 14: 717-724.

Zhang, L. P., Y. J. Zhang, W. J. Zhang, Q. J. Wu, B. Y. Xu, and D. Chu. 2005. Analysis of genetic diversity among different geographical populations and determination of biotypes of Bemisia tabaci in China. J. Appl. Entomol. 129: 121-128.

Received for publication 16 November 2006; accepted 26 March 2007. 